\title{
Further evidence of interaction between deprivation effects and stimulus control of responding: III
}

\author{
ROBERT W. POWELL and LINDA PALM \\ University of South Florida, Tampa, Florida $\$ 3620$
}

\begin{abstract}
Four pigeons were given baseline training under a multiple VI 60-sec/VI 60-sec schedule of food reinforcement. One component was correlated with a red key light to which a white light was added in order to signal the availability of a reinforcer. Under the other component, the key light was green at all times. This procedure resulted in few responses under the signaled component, except when the reinforcer was available, and consistent responding at high rates under the unsignaled component. Variations in hours of deprivation produced small increases in responding under the unsignaled component and no change under the signaled component. Systematic changes in body weight produced greater differential effects under the two components. Increases in deprivation resulted in increased responding during the unsignaled component, with little or no change in the rate of responding under the signaled component. The results suggest that when stimulus control results in low rates of response the influence of deprivation is diminished in relation to its effects upon behavior which is controlled at a high rate by a specific stimulus.
\end{abstract}

"Stimulus control refers to the extent to which the value of an antecedent stimulus determines the probability of occurrence of a conditioned response" (Terrace, 1966, p. 271). Stimulus control is described as existing on a continuum bounded by the terms "strong stimulus control" and "no stimulus control." The former term refers to a stimulus situation in which there is a high probability of occurrence of a particular class of response and a very low probability of all other classes of response. The latter term refers to a situation in which no particular response predominates in the presence of a particular stimulus. "Weak stimulus control," a commonly used term, is descriptive of a stimulus situation in which the probability of occurrence of one class of response is somewhat higher than all other response classes.

Discriminative control appears to exist as a subset under stimulus control, although the two terms have of ten been used interchangeably. Discriminative control is seen when a subject responds differentially in the presence of two or more stimuli. A typical procedure would consist of positive reinforcement correlated with one stimulus $\left(\mathrm{S}^{\mathrm{D}}\right)$ and extinction correlated with a second stimulus $\left(\mathrm{S}^{\Delta}\right)$. With sufficient training, this would result in the operant response occurring at a high rate in the presence of $S^{D}$ and at a low rate in the presence of $\mathrm{S}^{\Delta}$.

Research concerned with the influence of deprivation upon stimulus control has taken several approaches. One strategy has been to vary deprivation during gen-

Reprints may be obtained from Robert W. Powell, Department of Psychology, University of South Florida, Tampa, Florida 33620. eralization testing following nondifferential (generalization) training. Results of these experiments have been inconsistent, sometimes indicating that deprivation had little systematic effect upon generalization gradients (Kalish \& Haber, 1965; Newman \& Grice, 1965), while others have found nonmonotonic functional relationships (Thomas \& King, 1959). In several cases, generalization gradients have flattened as deprivation increased (Jenkins, Pascal, \& Walker, 1958; Kawashima, 1964), which probably conforms to intuitive expectation.

A second approach has been to vary deprivation following differential training (discrimination) in order to study any changes in responding in the presence of $S^{D}$ and $S^{\Delta}$. Some experimenters have found that increased rates under both $\mathrm{S}^{\mathrm{D}}$ and $\mathrm{S}^{\Delta}$ (Coate, 1964; Dinsmoor, 1952), but Gray (1965) found disproportionate increases in responding under $S^{\Delta}$, that is, the percentage increase in responding was much greater during extinction than during food reinforcement at high deprivation.

Our prior research in this area has taken a slightly different tack. Through differential training under two stimulus conditions, we have established different levels of discriminative control, typically within the same subject. Then we have manipulated deprivation in order to study how well discriminative control is maintained as a function of its preexisting level. We have found that the greater the accuracy of discrimination that developed during training, the smaller the effect of deprivation upon subsequent performance. When discrimination was inaccurate, increases in deprivation resulted in disproportionate increases in responding under $\mathrm{S}^{\Delta}$, as compared to increases under $S^{D}$. These findings 
suggest that as discriminative control of responding increases, performance becomes less and less susceptible to influence by deprivation (Powell, 1971, 1973).

In a similar vein, there have been several reports which demonstrate that drug effects are modulated by discriminative stimuli (Laties, 1972, 1975; Laties \& Weiss, 1966). Each of these studies found that behavior under strong external stimulus control was less apt to be influenced by a number of drugs, including amphetamine, scopolamine, and phenobarbital.

The present study was undertaken to replicate and extend our previous research, with concern for two main points: (1) establish and maintain stimulus control within one sensory modality and (2) establish differential response rates during the two schedule components, with equal rates of reinforcement in each component. With respect to the first point, our prior experiments had typically achieved strong and weak discriminative control in pigeons, through the use of visual and auditory stimuli, respectively. Thus, we wanted to eliminate this potential source of confounding. Also, in our earlier research, high response rates had been correlated with positive reinforcement, while low response rates were correlated with extinction. In the present study we wanted to assess the relative importance of response rate per se vs reinforcer availability in the interaction between deprivation effects and discriminative control of responding.

In order to achieve the goal of differential response rates in two schedule components having equal rates of reinforcement, we employed a multiple variableinterval (VI)/variable-interval (VI) schedule with signaled and unsignaled components. Under a conventional (unsignaled) VI schedule, the reinforcer is presented for the first response occurring after a specified time interval has elapsed, but the duration of the successive intervals varies unsystematically. A signaled VI schedule simply adds a distinct stimulus, such as a change in the key-light color, which occurs whenever a reinforcer becomes available for responding. Previous studies have shown that this procedure results in high and low response rates under the unsignaled and signaled components, respectively (Brownstein \& Hughes, 1970; Brownstein \& Newsom, 1970).

\section{METHOD}

\section{Subjects}

Four adult homing pigeons with extensive experimental histories were used. Water and grit were freely available to the birds in their home cages.

\footnotetext{
Apparatus

A Lehigh Valley pigeon test chamber, Model 1519C, was used. Purina Pigeon Checkers served as the reinforcer with a food hopper time of $2.0 \mathrm{sec}$ for each bird. The key light, which could be illuminated green, red, or white was turned off when food was presented. Data were recorded by digital counters and a Gerbrands cumulative recorder.
}

\section{Procedure}

Baseline training. Each bird was maintained at a stable weight during training. These weights ranged from $76 \%$ to $83 \%$ of free-feeding weight for the individual birds. The birds were exposed to a multiple schedule with equal-valued unsignaled VI 60 -sec and signaled VI 60 -sec components which alternated successively. During the unsignaled component, the key light was green. During the signaled component, the key light was red, but a white light was added to the key color whenever a reinforcer became available for responding. Thus, red was correlated with extinction, while white was correlated with a schedule of continuous reinforcement (CRF). Experimental sessions were $40 \mathrm{~min}$ in duration, consisting of two 10-min cycles each of the multiple schedule components. Baseline training continued until responding was stable under both multiple schedule components. The stability criterion was defined by variation in response rates within $\pm 15 \%$ of the mean rates over 10 consecutive sessions. Baseline training required 53, 44, 48, and 26 sessions for Pigeons 2, 23, 24, and 25, respectively.

Variation in hours of deprivation. Pigeons 23 and 25 were studied as deprivation was varied by withholding food for 24,48 , or $72 \mathrm{~h}$ prior to the experimental session. The usual sequence of deprivation periods was 72 (Monday), 24 (Tuesday), 48 (Thursday), and $24 \mathrm{~h}$ (Friday). Thus, each bird was normally studied 4 days/week. The birds were given supplemental feeding after each session in order to maintain body weight at approximately $83 \%$ of free-feeding weight. The number of sessions at each deprivation level for the two birds was as follows: Pigeon 23-72 h (7), $48 \mathrm{~h}$ (7), $24 \mathrm{~h}$ (9); Pigeon 25-72 h (9), 48 h (7), 24 h (17).

Systematic changes in body weight. All four birds were studied over a range of body weights, with a particular weight being maintained for 5 -day periods. Body weight was then changed by reduced or supplemental feeding for several days. Pigeons 3 and 24 were studied as body weight first increased and then decreased. For Pigeons 23 and 25 the reverse sequence was followed. In addition, each bird was food deprived for approximately $23 \mathrm{~h}$ prior to the experimental session.

\section{RESULTS AND DISCUSSION}

The two birds studied with variation in hours of deprivation both displayed strong discriminative control of responding at the conclusion of baseline training. Figure 1 shows that both birds responded at high rates under the unsignaled VI component and at very low rates under signaled VI. Under the latter component there was almost no responding except when the reinforcer was available. Consequently, response rates were approximately $1.0 / \mathrm{min}$, which corresponds to the average rate of reinforcement. Increases in deprivation resulted in slight to moderate increases in responding during the unsignaled VI component. Pigeon 23 showed a rate increase of approximately $10 \%$ from 24 to $48 \mathrm{~h}$, while Pigeon 25 showed an increase of roughly $20 \%$ from 24 to $72 \mathrm{~h}$ of deprivation. On the other hand, both birds showed no change in response rate during the signaled VI component as deprivation increased.

The two birds which were not studied with variation in hours of deprivation (Pigeons 3 and 24) also displayed strong discriminative control of responding, as shown in Figure 2. For these birds the baseline performance 


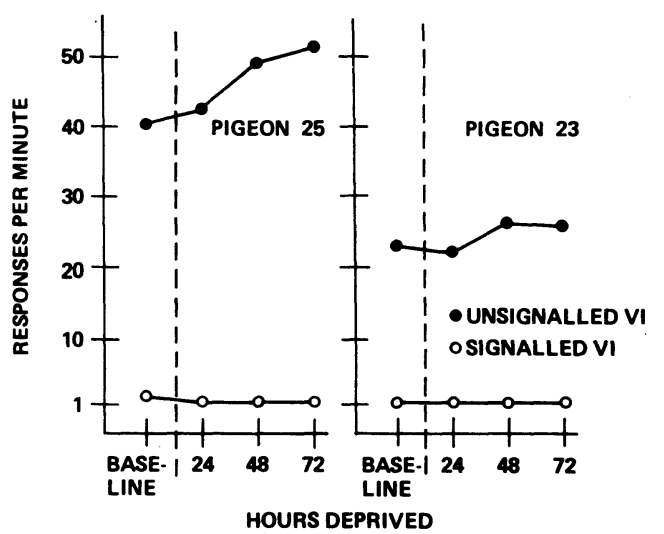

Figure 1. Mean response rates under signaled and unsignaled VI components for the two birds studied with variations in the hours of deprivation. Data points corresponding to baseline on the abscissa represent the mean performance over 10 sessions.

is presented as the initial weight level studied. The relationship between response rate and deprivation under the unsignaled VI component was consistent and orderly for each bird. Figure 2 shows that response rate increased as body weight decreased to approximately $80 \%$ of free-feeding weight. Then response rate curves remained essentially flat with further decreases in weight. These relationships were obtained independently of the sequence of weights studied. All four birds maintained an essentially constant response rate under the signaled VI component.

The present results appear consistent with our earlier findings (Powell, 1971, 1973), indicating that the matter of stimulus modality was not important. These findings also suggest that the differences in reinforcement density between components was not a critical factor in our earlier research.

Because of the procedural differences between these studies, it seems quite important to be very explicit in comparing behavioral effects and the conceptualizations derived from them. In the present experiment, for example, there was clearly strong discriminative control of responding, as seen in the differences in response rates under the two components. At the same time, there was strong stimulus control of responding at all times. The green key light controlled a high and steady rate of responding. The red key light controlled keypecking at essentially a zero rate. Red and white lights combined always resulted in an immediate keypeck.

As mentioned earlier, the signaled VI schedule could also be conceptualized as a mutliple CRF-extinction schedule. From this viewpoint, it would be inappropriate to make direct behavioral comparisons between the signaled and unsignaled components.

The problem encountered here is one familiar to most researchers. That is, the experimental question would be most easily resolved if one could produce differential behaviors under identical contingencies. This appears to be impossible. Consequently one must settle for contingencies which are as similar as possible. As a result, experimental findings are somewhat more difficult to interpret than would otherwise be the case.

Nevertheless, there appear to be two generalizations which are supported by the three studies we have so far conducted in this area. First, responding which occurs at a moderate or high rate in the presence of a specific stimulus will be directly influenced by variations in deprivation. Second, responding which is controlled at a very low rate by a specific stimulus will be uninfluenced by changes in deprivation. This is true even when the response occurs at a very high rate in the presence of other stimuli during the same experimental session.

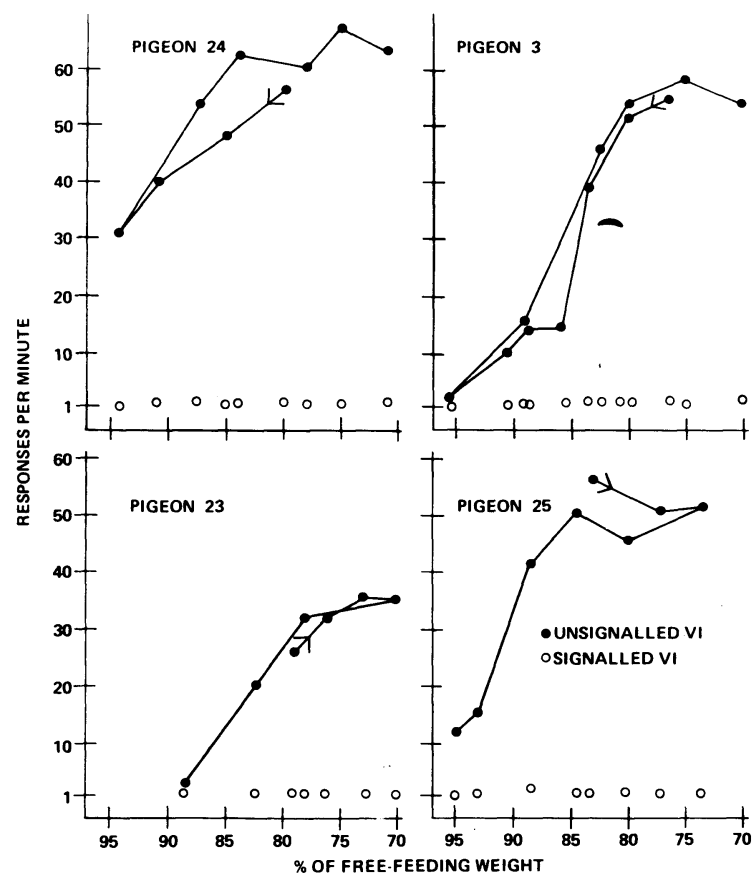

Figure 2. Mean response rates under signaled and unsignaled VI components for each bird as a function of body weight. An arrow appears next to the initial weight level studied and indicates the direction of subsequent weights investigated. The data points have been connected in the same sequence that the weight values were studied during the experiment. Each data point represents the mean performance over 5 sessions.

\section{REFERENCES}

Brownstein, A. J., \& Hughes, R. G. The role of response suppression in behavioral contrast: Signaled reinforcement. Psychonomic Science, 1970, 18, 50-52.

Brownstein, A. J., \& Newsom, C. Behavioral contrast in multiple schedules with equal reinforcement rates. Psychonomic Science, 1970, 18, 25-26.

Conte, W. B. Effect of deprivation on postdiscrimination stimulus generalization in the rat. Journal of Comparative and Physiological Psychology, 1964, 57, 134-138. 
Dinsmoor, J. A. The effect of hunger on discriminated responding. Journal of Abnormal and Social Psychology, 1952, 47, 67-72.

GRAY, J. A. Relations between stimulus intensity and operant response rate as a function of discrimination training and drive. Journal of Experimental Psychology, 1965, 69, 9-24.

Jenkins, W. O., Pascal, G. R., \& Walker, R. W., JR. Deprivation and generalization. Journal of Experimental Psychology, 1958, 56, 274-277.

Kalish, H. E., \& Haber, A. Prediction of discrimination from generalization following variations in deprivation level. Journal of Comparative and Physiological Psychology, $1965,60,125-128$.

Kawashima, T. The effect of drive on stimulus generalization. Japanese Psychological Research, 1964, 6, 88-95.

Laties, V. G. The modification of drug effects on behavior by external discriminative stimuli. Journal of Pharmacology and Experimental Therapeutics, 1972, 183, 1-13.

LAtIEs, V. G. The role of discriminative stimuli in modulating drug action. Federation Proceedings, 1975, 34, 1880-1888.

LAtIES, V. G., \& WEISS, B. Influence of drugs on behavior controlled by internal and external stimuli. Journal of Pharmacology and Experimental Therapeutics, 1966, 152, 388-396.

Newman, J. R., \& Grice, G. R. Stimulus generalization as a function of drive level, and the relation between two measures of response strength. Journal of Experimental Psychology, 1965, 69, 357-362.

Powell, R. W. Evidence of interaction between deprivation effects and stimulus control. Journal of Experimental Analysis of Behavior, 1971, 16, 95-104.

Powell, R. W. Effects of stimulus control and deprivation upon discriminative responding. Journal of the Experimental Analysis of Behavior, 1973, 19, 351-360.

Terrace, H. S. Stimulus control. In W. K. Honig (Ed.), Operant behavior: Areas of research and application. New York: Appleton-Century-Crofts, 1966.

Thомas, D. R., \& King, R. A. Stimulus generalization as a function of level of motivation. Journal of Experimental Psychology, 1959, 57, 323-328.

(Received for publication December 4, 1976.) 